\title{
Rapidly Destruction of the Hip Joint Associated with Enlarged Iliopsoas Bursa in a Patient with Refractory Rheumatoid Arthritis
}

\author{
Taro Yoshioka, Akitoshi Tachihara, Tairo Koyama, \\ Kayo Iwakawa, Manabu Sakane and Hiroshi Nakamura \\ Department of Joint Disease and Rheumatism, Nippon Medical School
}

\begin{abstract}
A case of refractory rheumatoid arthritis with a rapid destruction of the hip joint and an enlarged iliopsoas bursa is presented. Rapidly destructive coxarthrosis, chondrocalcinosis, suppurative arthritis, and pigmented villonodular synovitis were the differential diagnoses. Radiological examination showed that rheumatoid arthritis was most likely diagnosis. The patient was treated with total hip arthroplasty and etanercept, with good results.
\end{abstract}

(J Nippon Med Sch 2008; 75: 233-238)

Key words: rheumatoid arthritis, iliopsoas bursa, total hip arthroplasty

\section{Introduction}

An enlarged iliopsoas bursa is often associated with rheumatoid arthritis (RA). When associated hipjoint destruction is severe, suppurative arthritis and pigmented villonodular synovitis (PVS) should be excluded. Here, we report on a patient with refractory RA having rapidly destructive coxopathy associated with an enlarged iliopsoas bursa.

\section{Case Report}

A 45-year-old Japanese man with a 7-year history of RA complained of severe pain of the right hip joint. Disease activity had been high despite administration of betamethasone (1 $\mathrm{mg}$ daily) and methotrexate (12 mg weekly). He had discontinued the use of infliximab within 1 year because of the adverse effect of fever. Radiography showed collapse of the right femoral head with joint space loss (Fig. 1A). Three months later, he visited us again complaining of severe hip pain and leg-length discrepancy. Collapse of the femoral head and the acetabulum was also apparent (Fig. 1B). He was admitted for total hip arthroplasty (THA). Figure 1C shows a preoperative radiograph taken 5 months after the patient's first complaint.

Laboratory data at admission are shown in Table 1. The erythrocyte sedimentation rate was $84 \mathrm{~mm} / \mathrm{h}$ and the C-reactive protein level was $5.08 \mathrm{mg} / \mathrm{dL}$. The 28-joint disease activity score (DAS28) ${ }^{1}$ was 4.083, which suggested moderate disease activity. The right leg was shorter than the left by $2 \mathrm{~cm}$. The range of motion of the right hip joint was extremely limited, with severe motion pain. The patient could neither walk without the aid of bilateral crutches nor climb stairs. Body temperature was $38.6^{\circ} \mathrm{C}$. No groin mass was palpable and no local heat was noted around the hip. We considered the diagnoses of

Correspondence to Hiroshi Nakamura, MD, PhD, Department of Joint Disease and Rheumatism, Nippon Medical School, 1-1-5 Sendagi, Bunkyo-ku, Tokyo 113-8603, Japan

E-mail: nakamura@nms.ac.jp

Journal Website (http://www.nms.ac.jp/jnms/) 

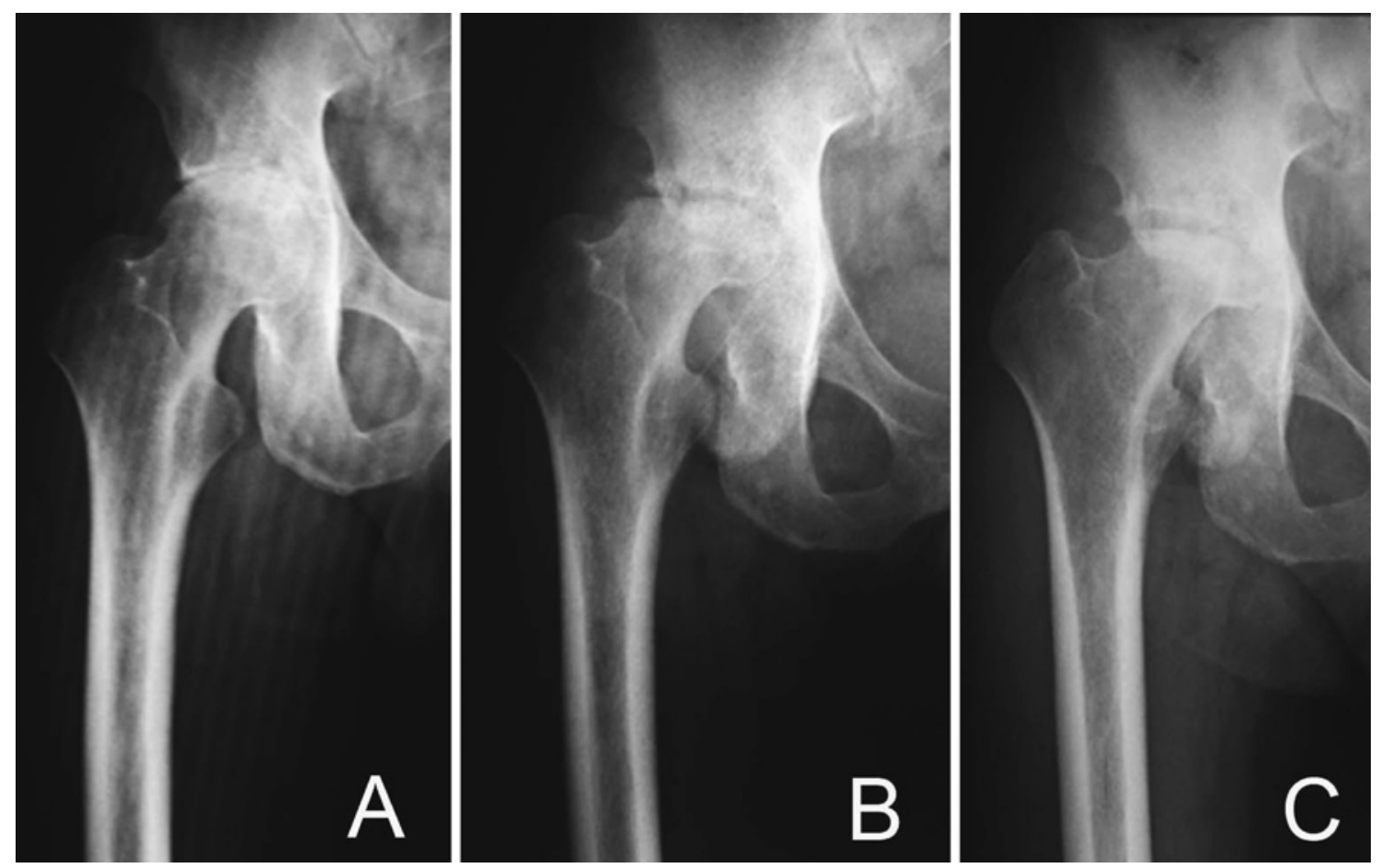

Fig. 1 Anteroposterior radiograph obtained at the time of first hip complaint (A), after the last outpatient visit (after 3 months) (B), and on admission (after 5 months) (C). Collapse of the femoral head progressed rapidly.

Table 1 Laboratory data at admission

\begin{tabular}{lclc}
\hline WBC & $10,700 / \mathrm{uL}$ & $\mathrm{ESR}$ & $84 \mathrm{~mm} / \mathrm{hr}$ \\
$\mathrm{RBC}$ & $422 \times 10^{4} / \mathrm{uL}$ & $\mathrm{CRP}$ & $5.07 \mathrm{mg} / \mathrm{dL}$ \\
$\mathrm{Hb}$ & $13.0 \mathrm{~g} / \mathrm{dL}$ & $\mathrm{RF}$ & $258 \mathrm{IU} / \mathrm{L}$ \\
$\mathrm{Ht}$ & $41.1 \%$ & $\mathrm{RAPA}$ & $640 \times$ \\
$\mathrm{GOT}$ & $16 \mathrm{IU} / \mathrm{L}$ & $\mathrm{MMP}-3$ & $205.1 \mathrm{ng} / \mathrm{mL}$ \\
$\mathrm{GPT}$ & $12 \mathrm{IU} / \mathrm{L}$ & & \\
$\mathrm{BUN}$ & $9.1 \mathrm{mg} / \mathrm{dL}$ & & \\
$\mathrm{Cre}$ & $0.43 \mathrm{mg} / \mathrm{dL}$ & & \\
\hline
\end{tabular}

iliopsoas bursa due to RA, PVS, suppurative arthritis, and malignant tumor.

Computed tomography (CT) showed severe joint destruction and masses anterior to the hip joint along with the iliopsoas muscle, which was associated with interior calcification and effusion (Fig. 2).

The lesion was composed of two parts. Magnetic resonance imaging (MRI) showed two enlarged masses anterior to the hip joint extending into the pelvis. The signal intensity of the lesion was lower than that of skeletal muscles on T1-weighted imaging (Fig. 3A). A T2-weighted image showed high signal intensity within the mass and suggested fluid collection (Fig. 3B). Axial views demonstrated the presence of two masses anterior to the joint, extending from the joint space. An anterior mass seemed to be located under the iliopsoas muscle and to extend into the pelvis (Fig. 4). The masses were not enhanced with gadolinium on T2-weighted imaging, and increased signal intensity was found in the periphery of the masses and inside the joint space on T1-weighted imaging and suggested synovial proliferation (Fig. 5).

Arthrocentesis and arthrography were performed. Yellowish serous fluid was aspirated, and arthrography demonstrated that the joint space was connected directly to the bursa, which had also been visualized with MRI (data not shown). No bacteria or mycobacteria were found to grow in the aspirated fluid and no crystals were found in the sediment. On the basis of these findings, we concluded that the lesion was an iliopsoas bursa with a rheumatoid hip. THA was performed without excision of the intrapelvic bursa, because a previous report have showen that iliopsoas bursitis resolves after THA $^{2}$. 
The joint cavity was filled with massive synovial tissue showing fibrinoid necrosis. A hole communicating with the iliopsoas bursa was found in the anterior capsule. Further exploration of the


Fig. 2 A CT scan shows masses anterior to the hip joint along with the psoas muscle associated with calcification and effusion [Remark 5] (arrows). (A) coronal view, (B) axial view. bursa was not performed for the reasons given above. The bone defect in the acetabulum was reinforced with an autologous bone graft (Fig. 6).

Histological examination of a synovial specimen demonstrated villous synovial proliferation with synovial cell proliferation, fibrinoid deposition, uptake of bone debris, lymph follicle formation, and fibrosis, which were consistent with active synovitis in RA (Fig. 7). Six weeks after the operation, disease activity was still high; The erythrocyte sedimentation rate was $64 \mathrm{~mm} / \mathrm{h}$, the C-reactive protein level was $3.0 \mathrm{mg} / \mathrm{dL}$, and DAS28 was 3.8 . Injection of etanercept was started to decrease disease activity. One year after the operation, RA was in remission, and no further enlargement of the iliopsoas bursa was found.

\section{Discussion}

The iliopsoas bursa is bound anteriorly by the iliopsoas muscle, posteriorly by the pectineal eminence and the capsule of hip joint, laterally by the iliofemoral ligament, and medially by the cotyloid ligament. Fourteen percent of the bursa communicates with the hip joint cavity, and retroperitoneal extension of the iliopsoas bursa sometimes occurs. When retroperitoneal extension does occur, the mass usually dissects anteriorly and superiorly along the iliopsoas muscle and enters the pelvis anterior to the muscle, with displacement and compression of adjacent pelvic structures ${ }^{3}$. A
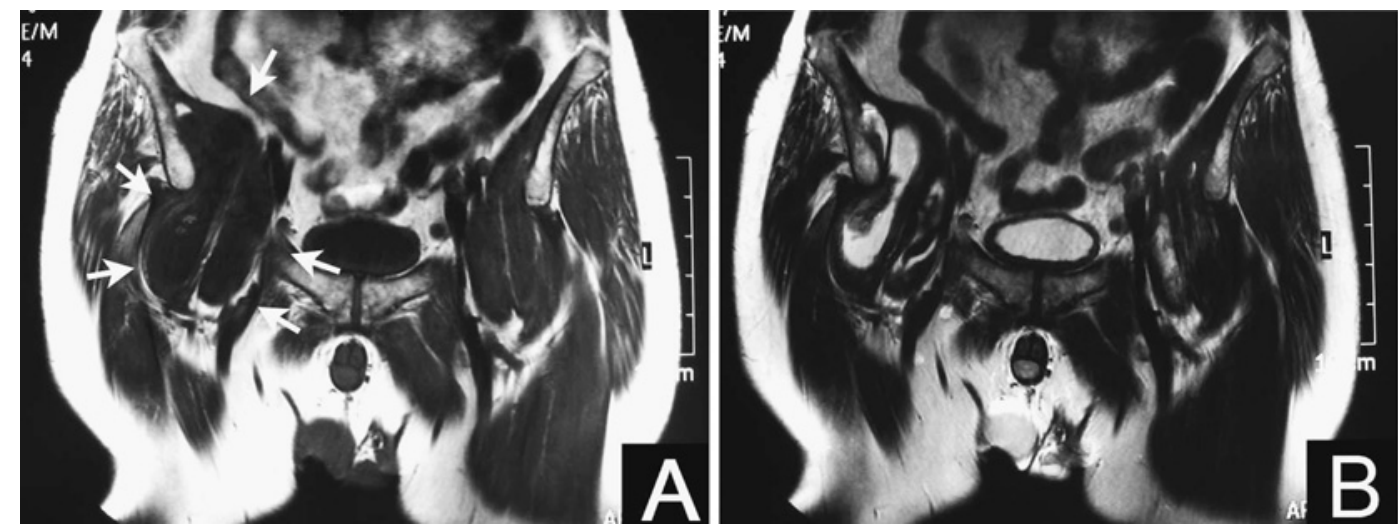

Fig. 3 (A) A T1-weighted MR image showing two enlarged masses anterior to the hip joint extending into the pelvis (arrows), the intensity of which is lower than that of skeletal muscles. (B) A T2weighted image shows high signal intensity within the mass, suggesting fluid collection. 

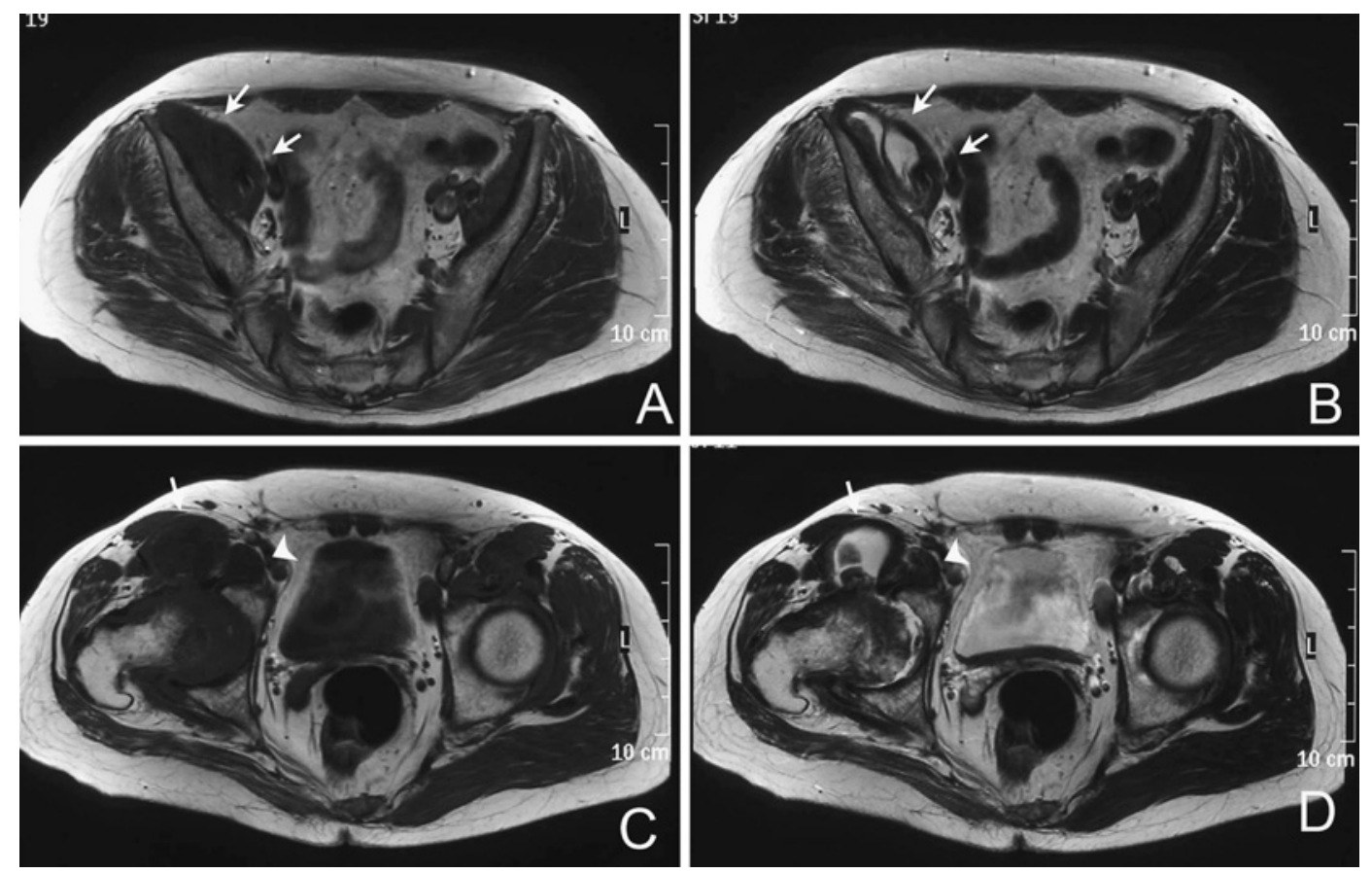

Fig. 4 Axial MR images at the pelvic level (A, B) and joint level (C, D). (A, C) T1-weighted images; (B, D) T2-weighted images. Two masses were found (arrow and arrowhead), and an anterior mass seemed to be located under the iliopsoas muscle and to extend into the pelvis.
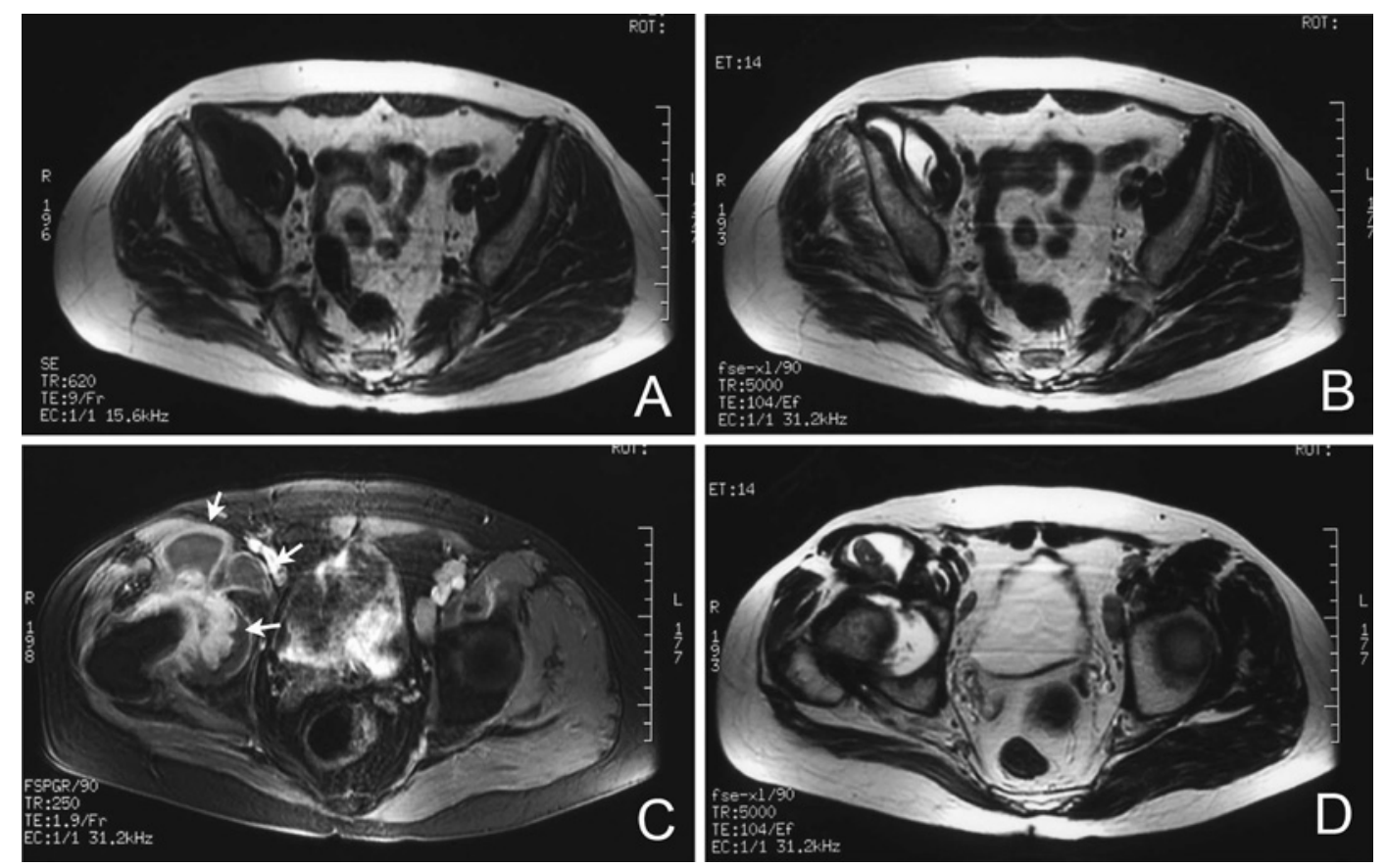

Fig. 5 Gadolinium-enhanced MR images at the pelvic level (A, B) and joint level (C, D). (A, C) T1weighted images; (B, D) T2-weighted images. Increased signal intensity is present in the periphery of the masses and inside the joint space (arrows) on the T1-weighted image (C).

syndrome has been described consisting of a palpable mass, extrinsic pressure on adjacent structures, such as the bowel, and radiographic changes in the hip characteristic of advanced degenerative or destructive arthritis ${ }^{3}$. In the present case, the peritoneal extension was found by chance 


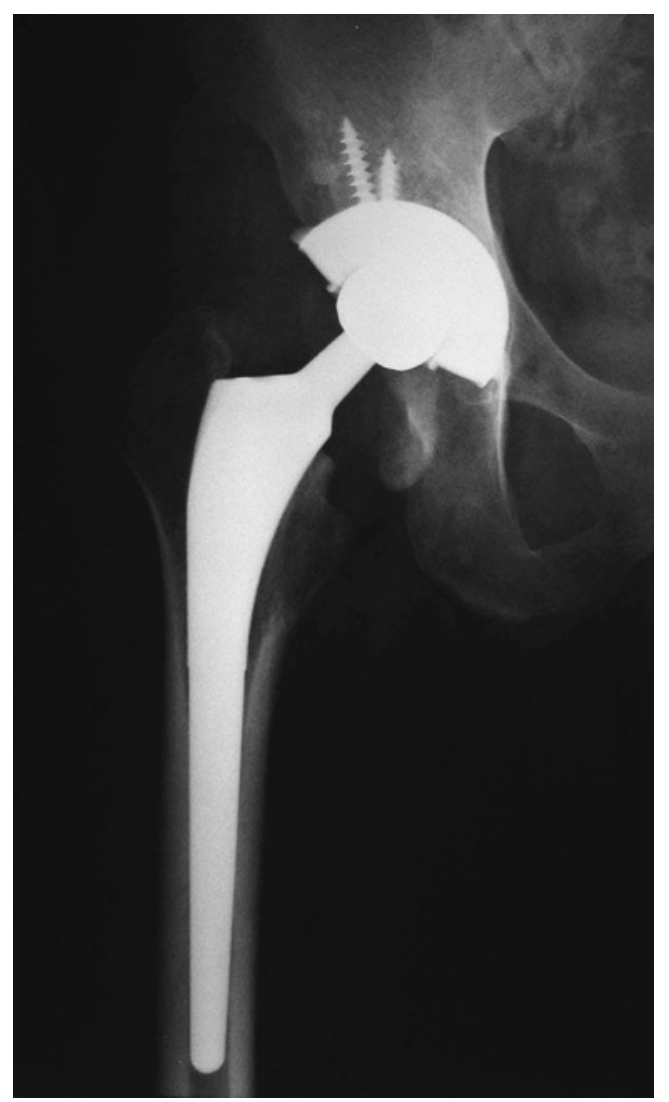

Fig. 6 THA was performed with an autologous bone graft in the acetabulum.

during preoperative imaging without any evidence of an enlarged iliopsoas bursa.

Rapid destruction of the hip joint is sometimes associated with rapidly destructive coxarthrosis (RDC), septic arthritis, and chondrocalcinosis as well as RA. RDC is a subset of osteoarthritis typically encountered in elderly women. Destruction of the femoral head and the acetabulum occurs within a few months after onset, with sclerosis and minimal or no osteophytes. This disease is characterized by painful disability with no significant reduction in joint motion. Although, the pathogenesis of RDC is not well understood, destruction of rheumatoid joints in this disease is caused by characteristic synovitis. The presence of calcification around the lesser trochanter on the anteroposterior radiographs and in the bursa on CT images suggests chondrocalcinosis. Chondrocalcinosis is induced by the deposition of calcium pyrophosphate dihydrate (CPPD) crystals and sometimes causes destructive arthritis ${ }^{6}$. However, no crystals were detected in the joint fluid

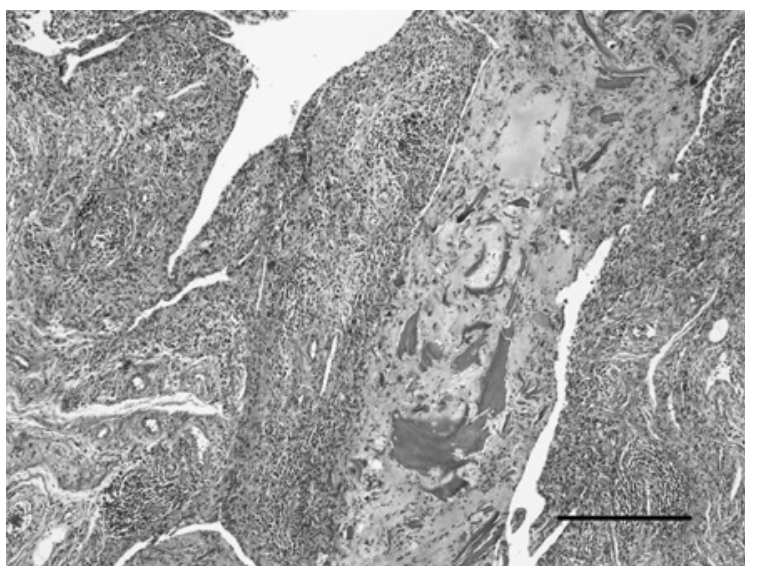

Fig. 7 Proliferated synovial tissue stained with hematoxylin and eosin. Synovial - cell proliferation, fibrinoid deposition, uptake of bone debris, lymph follicle formation, and fibrosis are present. Bar $=500 \mu \mathrm{m}$.

aspirated from the bursa, and CPPD crystals were not found on the histological examination.

PVS might have been the most important differential diagnosis in this case, as it was strongly suspected by a radiologist and might recur after excision of the synovium. Intra-articular PVS occurs in 2 per 1,000,000 persons and hip involvement is a rare, occurring in only $0.8 \%$ to $18 \%$ of cases of $\mathrm{PVS}^{7}$. The classic presentation of PVS of the hip is a monoarticular disease with progressive severity, limp, and stiffness. Radiographic findings of PVS include cyst formation in non-weight-bearing zones, femoral neck erosions, and joint-space narrowing. The key MRI finding is lobulated or nodular synovial proliferation containing hemosiderin with or without multiple bony erosions. Extension into the iliopsoas bursa is a known complication, resulting in an enlarging, nontender mass in the femoroinguinal region. In one case report, PVS extended from the hip joint as a large retroperitoneal mass, and the final diagnosis was made by mean of incisional biopsy ${ }^{10}$. The patient underwent THA and radical synovectomy; however, the large peritoneal component of the bursa was left in situ because of the extensive involvement of the pelvis with no progression 15 months after the operation ${ }^{10}$.

The radiographic pattern and the nature of aspirated fluid in our patient suggested that PVS was less likely, whereas destructive rheumatoid hip 
was most likely. The histological findings in the synovial tissue obtained during the operation were compatible with RA and did not show the foamy cells or hemosiderin-filled macrophages that are characteristic of PVS. In our patient, THA was performed with an excellent surgical result, and disease activity was suppressed by the use of etanercept, a biologic agent. To date the iliopsoas bursa has not re-enlarged.

In summary, a case of refractory RA with a rapid destruction of the hip joint and an enlarged iliopsoas bursa is presented. Although RDC, chondrocalcinosis, suppurative arthritis, and PVS were possible diagnoses, destructive rheumatoid hip was the most likely diagnosis. The patient was treated with THA and etanercept, with good results.

\section{References}

1. Prevoo ML, van't Hof MA, Kuper HH, van Leeuwen MA, van de Putte LB, van Riel PL: Modified disease activity scores that include twenty-eight-joint counts. Development and validation in a prospective longitudinal study of patients with rheumatoid arthritis. Arthritis Rheum 1995; 38: 44-48.

2. Matsumoto T, Jiji T, Mori T: Enlarged psoas muscle and iliopsoas bursitis associated with a rapidly destructive hip in a patient with rheumatoid arthritis. Mod Rheumatol 2006; 16: 52-54.

3. Melamed A, Bauer CA, Johnson JH: Iliopsoas bursal extension of arthritic disease of the hip. Radiology 1967; 89: 54-58.

4. Rosenberg ZS, Shankman S, Steiner GC, Kastenbaum DK, Norman A, Lazansky MG: Rapid destructive osteoarthritis: clinical, radiographic, and pathologic features. Radiology 1992; 182: 213-216.

5. Postel M, Kerboull M: Total prosthetic replacement in rapidly destructive arthrosis of the hip joint. Clin Orthop Relat Res 1970; 72: 138-144.

6. Menkes CJ, Simon F, Delrieu F, Forest M, Delbarre F: Destructive arthropathy in chondrocalcinosis articularis. Arthritis Rheum 1976; 19 (Suppl 3): 329348.

7. Gitelis S, Heligman D, Morton T: The treatment of pigmented villonodular synovitis of the hip. A case report and literature review. Clin Orthop Relat Res 1989; 239: 154-160.

8. Vastel L, Lambert P, De Pinieux G, Charrois O, Kerboull M, Courpied JP: Surgical treatment of pigmented villonodular synovitis of the hip. J Bone Joint Surg Am 2005; 87: 1019-1024.

9. Hughes TH, Sartoris DJ, Schweitzer ME, Resnick DL: Pigmented villonodular synovitis: MRI characteristics. Skeletal Radiol 1995; 24: 7-12.

10. Kallas KM, Vaughan L, Haghighi P, Resnick D: Pigmented villonodular synovitis of the hip presenting as a retroperitoneal mass. Skeletal Radiol 2001; 30: 469-474.

(Received, April 2, 2008)

(Accepted, April 18, 2008) 